\section{GW23-e1394 THE TREND AND VARIATION OF CARDIOVASCULAR DISEASE MORTALITY FROM YEAR OF 2004 TO 2010 IN CHINA}

doi:10.1136/heartjnl-2012-302920d.6

\author{
${ }^{1}$ Xiaofei Zhang, ${ }^{2}$ Dayi Hu, ${ }^{1}$ Xiaofei Zhang. ${ }^{1}$ National Center for Chronic and \\ Noncommunicable Disease Prevention and Control, China CDC; ${ }^{2}$ Department of \\ Cardiology, Peking University Peoples' Hospital
}

Objectives to report the epidemic status, trend and variation of cardiovascular disease (CVD) mortality from year of 2004 to 2010 in China.

Methods The mortality data album of population-based survey, 2004 and 2008 from National Disease Surveillance System are taken. This surveillance system is consisted by 161 disease surveillance points (DSPs) spreading in the 31 provinces of China; among them, 64 DSPs are in urban, 97 DSPs are in Rural. The area is divided into three regions: East, Central and West, geographically. The total population of surveillance is over 73 millions and it takes $6 \%$ around of the whole population, China. Cardiovascular disease included ischaemic heart Diseases (ICD10: I05-I09, I11, I20-I27, I30-I52) and cerebra-vascular disease (ICD-10: I60-I69). The epidemic status and trend of cardiovascular disease (CVD), cerebravascular disease (CD) and ischaemic heart disease (IHD) in mortalities from 2004 to 2008 are described by age, gender, urban/rural and region.

Results The mortality of CVD increased from 223.50/100 000 to $239.47 / 100000(+15.97 / 100000$ rise) in total, $229.06 / 100.000$ to $217.50 / 100000(-11.56 / 100000$ fall $)$ in urban, and 220.68/100 000 to $252.86 / 100000(+32.18 / 100000$ rise $)$ in rural. The similar trends were found in males and females among the total populations, urban populations and rural populations. However, the survey results showed that $\mathrm{CD}$ mortality decreased in urban and increased in rural. For example, the $\mathrm{CD}$ mortality rate fell from $134.76 / 100.000$ in 2004 to $131.58 / 100000$ ( $-3.18 / 100000$ fall) in 2010 in entire population, and 129.44/100 000 to $107.94 / 100000$ in urban $(-21.5 / 100000$ fall $)$, and rose from $137.44 / 100000$ to $145.97 / 100000$ (+8.53/100 000 rise) in rural. IHD mortality rates from 2004 to 2010 increased from $66.05 / 100000$ to 81.53 (+15.48/ 100000 rise) in entire population, $75.41 / 100000$ to $85.46 / 100000$ $(+10.05 / 100000$ rise) in urban, $61.32 / 100000$ to $79.69 / 100000$ $(+18.37 / 100000$ rise $)$ in rural. No mater what CD or IHD, the mortality rates are dramatically more increase in rural populations than urban populations. The similar variation and trend were found in males and females among entire, urban and rural populations. The age-standardised mortality rates and variations of CVD, $\mathrm{CD}$ and IHD showed the similar trend as true crude mortality rates in 2004 and 2010. The surveillance results also demonstrated that the rates of CVD mortality increased from 223.5/100.000 in 2004 to $230.24 / 1000002005$, then turned down to the lowest point at $54.91 / 100000$ in 2006 , then rose again continually year by year until reached the highest rate of $81.53 / 100000$ in 2010. There are similar trends and variations for $\mathrm{CD}$ and IHD both in crude mortality rates and age-standardised mortality rates among males and females. However, it is worthwhile to notice that the mortality rates for $\mathrm{CVD}, \mathrm{CD}$ and $\mathrm{IHD}$ among rural populations increased so faster, even exceeded the rates of urban populations. For example, for $\mathrm{CD}$, the rate in rural exceeded it in urban after year of 2006 among all, males and females; for IHD, the rate in rural continued to rise and were close to the rate in urban in year 2010 among all, males and females.

Conclusions The mortality of CVD, CD and IHD from 2004 to 2010 continued to increase except year of 2006 although there were waves between year 2004 and 2006. The age-standardised mortality rates of CVD, CD and IHD showed the similar trend and variations both for males and females. But the sharp faster speed of mortality rates in CVD, CD and IHD were from rural populations. More and more usage of advanced new medical techniques or devices, medication and medical services would decrease CVD mortality and increase CVD prevalence, particularly in urban area. Meanwhile, increase of CVD mortality would be explained by additional results of behaviour and risk factor survey for non-communicable and chronic disease in China-high levels or high rates of CVD risk factors, unhealthy lifestyle and behaviours besides the availability of accessing health care in the advanced medical therapies and techniques, especially for rural populations. The implementation should be strengthened and taken more at the CVD primary prevention level besides the aspect of CVD secondary prevention level, particularly in rural areas. 